\title{
HARDY TYPE INEQUALITIES \\ RELATED TO CARNOT-CARATHÉODORY DISTANCE ON THE HEISENBERG GROUP
}

\author{
QIAO-HUA YANG \\ (Communicated by Mei-Chi Shaw)
}

\begin{abstract}
Being motivated by a representation formula associated with the Korányi-Folland nonisotropic gauge proved by Cohn and $\mathrm{Lu}$, we prove an analogous representation formula related to the Carnot-Carathéodory distance on the Heisenberg group. Using this formula, we obtain some Hardy inequalities associated with the Carnot-Carathéodory distance on such groups.
\end{abstract}

\section{INTRODUCTION}

It is well known that Hardy's inequality and its generalization in $\mathbb{R}^{\mathbb{N}}$ play an important role in many areas of mathematics. In the case of the Heisenberg group $\mathbb{H}_{n}$, Garofalo and Lanconelli (cf. [9]) first proved the following Hardy inequality:

$$
\int_{\mathbb{H}_{n}}\left|\nabla_{H} u\right|^{2} \geq \frac{(Q-2)^{2}}{4} \int_{\mathbb{H}_{n}} \frac{u^{2}}{d^{2}}\left|\nabla_{H} d\right|^{2}, \quad u \in C_{0}^{\infty}\left(\mathbb{H}_{n} \backslash\{e\}\right),
$$

where $e$ is the origin of $\mathbb{H}_{n}, d=\left(|z|^{4}+t^{2}\right)^{\frac{1}{4}}$ is the Korányi-Folland nonisotropic gauge induced by the fundamental solution and $Q=2 n+2$ is the homogeneous dimension of $\mathbb{H}_{n}$ (see also [10]). P. Niu et al. ([12]) generalized inequality (1.1) to the nonlinear cases. That is, for $1<p<Q$, we have

$$
\int_{\mathbb{H}_{n}}\left|\nabla_{H} u\right|^{p} \geq\left(\frac{Q-p}{p}\right)^{p} \int_{\mathbb{H}_{n}} \frac{|u|^{p}}{d^{p}}\left|\nabla_{H} d\right|^{p}, u \in C_{0}^{\infty}\left(\mathbb{H}_{n} \backslash\{e\}\right) .
$$

To obtain (1.2), they used a Picone type identity on $\mathbb{H}_{n}$. D'Ambrosio (cf. 3, 4, 5]) extended (1.2) to some degenerate elliptic differential operators such as the Heisenberg-Greiner operator, a Baouendi-Grushin type operator and the subLaplacian on Carnot groups. More recently, Danielli, Garofalo and Phuc (cf. [6, 7]) consider various types of Hardy-Sobolev inequalities on a Carnot-Carathéodory space. They use the fundamental solution of the corresponding $p$-Laplace operator and generalize (1.2) to Carnot groups of arbitrary step.

The aim of this paper is to prove an analogous Hardy-type inequality (1.2) on $\mathbb{H}_{n}$, where the Korányi-Folland nonisotropic gauge is replaced by the CarnotCarathéodory distance $d_{c c}$ on $\mathbb{H}_{n}$ (we refer to [1, 11] for more information about

Received by the editors September 10, 2009 and, in revised form, September 2, 2010 and June 14, 2011.

2010 Mathematics Subject Classification. Primary 22E25; Secondary 26D10.

Key words and phrases. Hardy inequalities, Heisenberg group, Carnot-Carathéodory distance.

This work was supported by the National Science Foundation of China under Grant No. 10671009. 
this distance). To our knowledge, little is known about Hardy inequalities related to such a distance. Since $d_{c c}$ is not differentiable in the center of $\mathbb{H}_{n}$, it seems that the methods used in [3, 5, 7, 12, do not work for such a distance. To do so, we prove a representation formula associated with $d_{c c}$, and the idea is due to Cohn and Lu ([2]). The main result is the following theorem.

Theorem 1.1. Let $1<p<Q$ and $u \in C_{0}^{\infty}\left(\mathbb{H}_{n}\right)$. Then

$$
\int_{\mathbb{H}_{n}}\left|\nabla_{H} u\right|^{p} \geq\left(\frac{Q-p}{p}\right)^{p} \int_{\mathbb{H}_{n}} \frac{|u|^{p}}{d_{c c}^{p}} .
$$

The Hardy type inequalities with weights imply the following Hardy-Rellich type inequalities (see e.g. [14] for analogous inequalities on $\mathbb{R}^{N}$ ).

Theorem 1.2. Let $\mathbb{Z}=\left\{(z, t) \in \mathbb{H}_{n}: z=0\right\}$ be the center of $\mathbb{H}_{n}$. It follows, for all $u \in C_{0}^{\infty}\left(\mathbb{H}_{n} \backslash \mathbb{Z}\right)$, that

$$
\int_{\mathbb{H}_{n}}\left|\Delta_{H} u\right|^{2} \geq\left(\frac{Q(Q-4)}{4}\right)^{2} \int_{\mathbb{H}_{n}} \frac{u^{2}}{d_{c c}^{4}}, \quad n \geq 2,
$$

and

$$
\int_{\mathbb{H}_{n}}\left|\Delta_{H} u\right|^{2} \geq \frac{Q^{2}}{4} \int_{\mathbb{H}_{n}} \frac{\left|\nabla_{H} u\right|^{2}}{d_{c c}^{2}}, \quad n \geq 3
$$

Finally, we obtain some Hardy inequalities involving the distance from the boundary which generalize a result of D'Ambrosio (cf. [5]).

Theorem 1.3. Let $p>1$ and $u \in C_{0}^{\infty}\left(B_{c c}(e, \rho)\right)$, where $B_{c c}(e, \rho)$ is the CarnotCarathéodory metric ball centered at the origin. Then

$$
\int_{B_{c c}(e, \rho)}\left|\nabla_{H} u\right|^{p} \geq\left(\frac{p-1}{p}\right)^{p} \int_{B_{c c}(e, \rho)} \frac{|u|^{p}}{\left(\rho-d_{c c}\right)^{p}} .
$$

\section{Notation AND PRELIMINARIES}

Let $\mathbb{H}_{n}=\left(\mathbb{C}^{n} \times \mathbb{R}, \circ\right)$ be the the $(2 n+1)$-dimensional Heisenberg group whose group structure is given by

$$
(z, t) \circ\left(z^{\prime}, t^{\prime}\right)=\left(z+z^{\prime}, t+t^{\prime}+2 \operatorname{Im}\left\langle z, z^{\prime}\right\rangle\right)
$$

where $z=\left(z_{1}, \cdots, z_{n}\right), z^{\prime}=\left(z_{1}^{\prime}, \cdots, z_{n}^{\prime}\right) \in \mathbb{C}^{n}, z_{j}=x_{j}+i y_{j}\left(x_{j}, y_{j} \in \mathbb{R}\right)$ and $\left\langle z, z^{\prime}\right\rangle=\sum_{j=1}^{n} z_{j} \cdot \overline{z_{j}}$. The vector fields

$$
X_{j}=\frac{\partial}{\partial x_{j}}+2 y_{j} \frac{\partial}{\partial t}, \quad Y_{j}=\frac{\partial}{\partial y_{j}}-2 x_{j} \frac{\partial}{\partial t}
$$

$(j=1, \cdots, n)$ are left invariant and generate the Lie algebra of $\mathbb{H}_{n}$. The commutators of these vector fields satisfy

$$
\left[X_{j}, Y_{j}\right]=-4 \frac{\partial}{\partial t}, \quad j=1,2, \cdots, n
$$


with all other brackets equal to zero. We denote by $\mathbb{Z}=\left\{(z, t) \in \mathbb{H}_{n}: z=0\right\}$ the center of $\mathbb{H}_{n}$. Then Kohn's sub-Laplacian on $\mathbb{H}_{n}$ is

$$
\Delta_{H}=\sum_{j=1}^{n}\left(X_{j}^{2}+Y_{j}^{2}\right)=\sum_{j=1}^{n}\left(\frac{\partial^{2}}{\partial x_{j}^{2}}+\frac{\partial^{2}}{\partial y_{j}^{2}}\right)+4|z|^{2} \frac{\partial^{2}}{\partial t^{2}}+4 \sum_{k=1}^{n}\left(y_{j} \frac{\partial}{\partial x_{j}}-x_{j} \frac{\partial}{\partial y_{j}}\right) \frac{\partial}{\partial t}
$$

and the horizontal gradient is the $(2 n)$-dimensional vector given by

$$
\nabla_{H}=\left(X_{1}, \cdots, X_{n}, Y_{1}, \cdots, Y_{n}\right) .
$$

We call a curve $\gamma:[a, b] \rightarrow \mathbb{H}_{n}$ a horizontal curve connecting two points $\xi, \eta \in \mathbb{H}_{n}$ if $\gamma(a)=\xi, \gamma(b)=\eta$ and $\gamma^{\prime}(s) \in \operatorname{span}\left\{X_{1}, \cdots, X_{n}, Y_{1}, \cdots, Y_{n}\right\}$ for all $s$. Then the Carnot-Carathéodory distance between $\xi, \eta$ is defined as

$$
d_{c c}(\xi, \eta)=\inf _{\gamma} \int_{a}^{b}\left\|\gamma^{\prime}(s)\right\| d s
$$

where the infimum is taken over all horizontal curves $\gamma$ connecting $\xi$ and $\eta$. It is known that any two points $\xi, \eta$ on $\mathbb{H}_{n}$ can be joined by a horizontal curve of finite length and then $d_{c c}$ is a metric on $\mathbb{H}_{n}$. An important feature of this distance function is that the distance and thus the associated metric balls are left-invariant. With this norm, we can define the metric ball centered at the origin and with radius $\rho$ associated with this metric by

$$
B_{c c}(e, \rho)=\left\{\eta: d_{c c}(e, \eta)<\rho\right\}
$$

and the unit sphere $\Sigma=\partial B_{c c}(e, 1)$. For simplicity, we write $d_{c c}(\xi)=d_{c c}(e, \xi)$.

For each real number $\lambda>0$, there is a dilation naturally associated with the group structure which is usually denoted as $\delta_{\lambda}(z, t)=\left(\lambda z, \lambda^{2} t\right)$. The Jacobian determinant of $\delta_{\lambda}$ is $\lambda^{Q}$, where $Q=2 n+2$ is the homogeneous dimension of $H_{n}$. For simplicity, we use the notation $\lambda(z, t)=\left(\lambda z, \lambda^{2} t\right)$. The Carnot-Carathéodory distance $d_{c c}$ satisfies

$$
d_{c c}(\lambda(z, t))=\lambda d_{c c}(z, t), \quad \lambda>0 .
$$

Given any $\xi=(z, t) \in \mathbb{H}_{n}$, set $z^{*}=\frac{z}{d_{c c}(z, t)}, t^{*}=\frac{t}{d_{c c}(z, t)^{2}}$ and $\xi^{*}=\left(z^{*}, t^{*}\right)$. The polar coordinates on $\mathbb{H}_{n}$ associated with $d_{c c}$ are the following (cf. [8], Proposition 1.15 or [1], via the coarea formula):

$$
\int_{\mathbb{H}_{n}} f(z, t) d x d t=\int_{0}^{\infty} \int_{\Sigma} f\left(\lambda\left(z^{*}, t^{*}\right)\right) \lambda^{Q-1} d \sigma d \lambda
$$

for all $f \in L^{1}\left(\mathbb{H}_{n}\right)$.

Set

$$
\mu(\theta)=\frac{2 \theta-\sin 2 \theta}{2 \sin ^{2} \theta}:(-\pi, \pi) \rightarrow \mathbb{R} .
$$

$\mu$ is a diffeomorphism of the interval $(-\pi, \pi)$ onto $\mathbb{R}$ (cf. [1]). We denote by $\mu^{-1}$ the inverse function of $\mu$. The Carnot-Carathéodory distance $d_{c c}$ satisfies (cf. [1] )

$$
d_{c c}(z, t)= \begin{cases}\pi|t|, & z=0 \\ \frac{\theta}{\sin \theta}\|z\|, & z \neq 0\end{cases}
$$

where

$$
\theta=\mu^{-1}\left(\frac{t}{\|z\|^{2}}\right) \quad \text { and } \quad\|z\|^{2}=\sum_{j=1}^{n}\left\|z_{j}\right\|^{2}
$$


On the other hand, if we set

$$
\Omega=\left\{\left(B_{1}, \cdots, B_{n}, A_{1}, \cdots, A_{n}, \phi, \rho\right): \sum_{j=1}^{n}\left(A_{j}^{2}+B_{j}^{2}\right)=1,-2 \pi \leq \phi \rho \leq 2 \pi, \rho \geq 0\right\}
$$

and define $\Phi: \Omega \rightarrow \mathbb{H}_{n}$ by $\Phi\left(B_{1}, \cdots, B_{n}, A_{1}, \cdots, A_{n}, \phi, \rho\right)=(z, t)$, where

$$
\left\{\begin{array}{l}
x_{j}\left(B_{1}, \cdots, B_{n}, A_{1}, \cdots, A_{n}, \phi, \rho\right)=\frac{A_{j}(1-\cos \phi \rho)+B_{j} \sin \phi \rho}{\phi} \\
y_{j}\left(B_{1}, \cdots, B_{n}, A_{1}, \cdots, A_{n}, \phi, \rho\right)=\frac{-B_{j}(1-\cos \phi \rho)+A_{j} \sin \phi \rho}{\phi} \\
t\left(B_{1}, \cdots, B_{n}, A_{1}, \cdots, A_{n}, \phi, \rho\right)=2 \frac{\phi \rho-\sin \phi \rho}{\phi^{2}}
\end{array}\right.
$$

$(j=1,2, \cdots, n)$, then the range of $\Phi$ is $\mathbb{H}_{n}$ and the center $\mathbb{Z}$ is just the set of points $\Phi\left(B_{1}, \cdots, B_{n}, A_{1}, \cdots, A_{n}, \phi, \rho\right)$ with $\phi \rho= \pm 2 \pi$. Furthermore, if one fixes $\rho>0$, equation (2.3) parameterizes $\partial B_{c c}(e, \rho)$ (cf. [1]).

From (2.3), we have

$$
\|z\|=\sqrt{\frac{2(1-\cos \phi \rho)}{\phi^{2}}}=\sqrt{\frac{4 \sin ^{2} \frac{\phi \rho}{2}}{\phi^{2}}}=\frac{2 \sin \frac{\phi \rho}{2}}{\phi}
$$

and

$$
\mu(\theta)=\frac{t}{\|z\|^{2}}=\frac{\phi \rho-\sin \phi \rho}{2 \sin ^{2} \frac{\phi \rho}{2}}=\mu\left(\frac{\phi \rho}{2}\right) .
$$

Therefore,

$$
\theta=\frac{\phi \rho}{2} \quad \text { when }-2 \pi<\phi \rho<2 \pi
$$

since $\mu$ is a a diffeomorphism of the interval $(-\pi, \pi)$ onto $\mathbb{R}$.

\section{MAin RESUlts}

To prove the main result, we first need the following representation formula, and the basic idea of the proof is the same as Cohn and Lu's (see [2]).

Lemma 3.1. Let $R_{2}>R_{1}>0$ and $f \in C^{1}\left(B_{c c}\left(e, R_{2}\right) \backslash B_{c c}\left(e, R_{1}\right)\right)$. Then

$$
\int_{\Sigma} f\left(R_{2} \xi^{*}\right) d \sigma-\int_{\Sigma} f\left(R_{1} \xi^{*}\right) d \sigma=\int_{B_{c c}\left(e, R_{2}\right) \backslash B_{c c}\left(e, R_{1}\right)}\left\langle\nabla_{H} f, \nabla_{H} d_{c c}\right\rangle \cdot \frac{1}{d_{c c}^{Q-1}} d \xi
$$

Proof. Let $\xi^{*}$ be a point on the sphere, that is, $\xi^{*}=\left(z^{*}, t^{*}\right)$, where $d_{c c}\left(z^{*}, t^{*}\right)=$ 1. We consider for $0<R_{1}<R_{2}$ the following difference using the fundamental theorem of calculus:

$$
\begin{aligned}
& \int_{\Sigma} f\left(R_{2} \xi^{*}\right) d \sigma-\int_{\Sigma} f\left(R_{1} \xi^{*}\right) d \sigma=\int_{\Sigma} \int_{R_{1}}^{R_{2}} \frac{d}{d \rho} f\left(\rho \xi^{*}\right) d \rho d \sigma \\
& =\int_{\Sigma} \int_{R_{1}}^{R_{2}}\left(\sum_{j=1}^{n} \frac{\partial f(\xi)}{\partial x_{j}} \cdot \frac{\partial x_{j}}{\partial \rho}+\sum_{j=1}^{n} \frac{\partial f(\xi)}{\partial y_{j}} \cdot \frac{\partial y_{j}}{\partial \rho}+\frac{\partial f(\xi)}{\partial t} \cdot \frac{\partial t}{\partial \rho}\right) d \rho d \sigma
\end{aligned}
$$


where $\xi=(x, t)=\rho \xi^{*}$. Using equation (2.3), we have

$$
\begin{aligned}
& \sum_{j=1}^{n} \frac{\partial f(\xi)}{\partial x_{j}} \cdot \frac{\partial x_{j}}{\partial \rho}+\sum_{j=1}^{n} \frac{\partial f(\xi)}{\partial y_{j}} \cdot \frac{\partial y_{j}}{\partial \rho}+\frac{\partial f(\xi)}{\partial t} \cdot \frac{\partial t}{\partial \rho} \\
& =\sum_{j=1}^{n} \frac{\partial f(\xi)}{\partial x_{j}} \cdot\left(A_{j} \sin \phi \rho+B_{j} \cos \phi \rho\right)+\sum_{j=1}^{n} \frac{\partial f(\xi)}{\partial y_{j}} \cdot\left(-B_{j} \sin \phi \rho+A_{j} \cos \phi \rho\right) \\
& +\frac{\partial f(\xi)}{\partial t} \cdot \frac{2-2 \cos \phi \rho}{\phi} \\
& =\sum_{j=1}^{n}\left[X_{j} f \cdot\left(A_{j} \sin \phi \rho+B_{j} \cos \phi \rho\right)+Y_{j} f \cdot\left(-B_{j} \sin \phi \rho+A_{j} \cos \phi \rho\right)\right]+\frac{\partial f(\xi)}{\partial t} \\
& \quad \cdot\left\{\frac{2-2 \cos \phi \rho}{\phi}-\sum_{j=1}^{n}\left[2 y_{j}\left(A_{j} \sin \phi \rho+B_{j} \cos \phi \rho\right)-2 x_{j}\left(-B_{j} \sin \phi \rho+A_{j} \cos \phi \rho\right)\right]\right\} .
\end{aligned}
$$

Again using (2.3), we have

$$
\begin{aligned}
\sum_{j=1}^{n}[ & \left.2 y_{j}\left(A_{j} \sin \phi \rho+B_{j} \cos \phi \rho\right)-2 x_{j}\left(-B_{j} \sin \phi \rho+A_{j} \cos \phi \rho\right)\right] \\
= & 2 \sum_{j=1}^{n} \frac{\left(-B_{j}(1-\cos \phi \rho)+A_{j} \sin \phi \rho\right)\left(A_{j} \sin \phi \rho+B_{j} \cos \phi \rho\right)}{\phi} \\
& \quad-2 \sum_{j=1}^{n} \frac{\left(A_{j}(1-\cos \phi \rho)+B_{j} \sin \phi \rho\right)\left(-B_{j} \sin \phi \rho+A_{j} \cos \phi \rho\right)}{\phi} \\
= & 2 \sum_{j=1}^{n} \frac{\left(A_{j}^{2}+B_{j}^{2}\right)(1-\cos \phi \rho)}{\phi}=2 \frac{1-\cos \phi \rho}{\phi} .
\end{aligned}
$$

Combining (3.2), (3.3) and (3.4), we obtain

$$
\begin{aligned}
& \int_{\Sigma} f\left(R_{2} \xi^{*}\right) d \sigma-\int_{\Sigma} f\left(R_{1} \xi^{*}\right) d \sigma \\
& =\int_{\Sigma} \int_{R_{1}}^{R_{2}}\left[X_{j} f \cdot\left(A_{j} \sin \phi \rho+B_{j} \cos \phi \rho\right)+Y_{j} f \cdot\left(-B_{j} \sin \phi \rho+A_{j} \cos \phi \rho\right)\right] d \rho d \sigma .
\end{aligned}
$$

Rewriting the last expression into a solid integral using the polar coordinates over $\mathbb{H}_{n}$, we get

$$
\begin{aligned}
& \int_{\Sigma} f\left(R_{2} \xi^{*}\right) d \sigma-\int_{\Sigma} f\left(R_{1} \xi^{*}\right) d \sigma \\
& =\int_{B_{c c}\left(e, R_{2}\right) \backslash B_{c c}\left(e, R_{1}\right)} \sum_{j=1}^{n} \frac{\left(A_{j} \sin \phi \rho+B_{j} \cos \phi \rho\right) X_{j} f+\left(-B_{j} \sin \phi \rho+A_{j} \cos \phi \rho\right) Y_{j} f}{d_{c c}(\xi)^{Q-1}} d \xi .
\end{aligned}
$$

To finish our proof, it is enough to show that

$$
X_{j} d_{c c}(\xi)=A_{j} \sin \phi \rho+B_{j} \cos \phi \rho, \quad Y_{j} d_{c c}(\xi)=-B_{j} \sin \phi \rho+A_{j} \cos \phi \rho
$$

$(j=1, \cdots, n)$ in $\mathbb{H}_{n} \backslash \mathbb{Z}$. This is done by the following Lemma 3.2. The proof of Lemma 3.1 is now completed. 
Lemma 3.2. It follows that, for $\xi=(z, t) \in \mathbb{H}_{n} \backslash \mathbb{Z}$,

$$
X_{j} d_{c c}(\xi)=A_{j} \sin \phi \rho+B_{j} \cos \phi \rho, \quad Y_{j} d_{c c}(\xi)=-B_{j} \sin \phi \rho+A_{j} \cos \phi \rho
$$

$(j=1, \cdots, n)$.

Proof. Recall that if $z \neq 0$, then

$$
d_{c c}(\xi)=d_{c c}(z, t)=\frac{\theta}{\sin \theta}\|z\|,
$$

where $\theta=\mu^{-1}\left(t /\|z\|^{2}\right)$. A simple calculation shows that, for $j=1, \cdots, n$,

$$
\begin{gathered}
\mu^{\prime}(\theta)=\frac{2 \sin \theta-2 \theta \cos \theta}{\sin ^{3} \theta}, \quad \frac{\partial \theta}{\partial x_{j}}=-\frac{2 t x_{j}}{\|z\|^{4}} \cdot \frac{1}{\mu^{\prime}(\theta)}=-\frac{t x_{j}}{\|z\|^{4}} \cdot \frac{\sin ^{3} \theta}{\sin \theta-\theta \cos \theta}, \\
\frac{\partial \theta}{\partial y_{j}}=-\frac{t y_{j}}{\|z\|^{4}} \cdot \frac{\sin ^{3} \theta}{\sin \theta-\theta \cos \theta}, \quad \frac{\partial \theta}{\partial t}=\frac{1}{\|z\|^{2}} \cdot \frac{\sin ^{3} \theta}{2 \sin \theta-2 \theta \cos \theta} .
\end{gathered}
$$

Therefore, for $\xi=(z, t) \in \mathbb{H}_{n} \backslash \mathbb{Z}$,

$$
\begin{aligned}
\frac{\partial d_{c c}(\xi)}{\partial x_{j}} & =\frac{\partial}{\partial x_{j}}\left(\frac{\theta}{\sin \theta}\|z\|\right)=\frac{x_{j}}{\|z\|} \cdot \frac{\theta}{\sin \theta}+\|z\| \cdot \frac{\sin \theta-\theta \cos \theta}{\sin ^{2} \theta} \cdot \frac{\partial \theta}{\partial x_{j}} \\
& =\frac{x_{j}}{\|z\|} \cdot \frac{\theta}{\sin \theta}-\frac{t x_{j}}{\|z\|^{3}} \cdot \sin \theta=\frac{x_{j}}{\|z\|} \cdot \frac{\theta}{\sin \theta}-\frac{x_{j}}{\|z\|} \cdot \mu(\theta) \sin \theta \\
& =\frac{x_{j}}{\|z\|} \cdot \cos \theta .
\end{aligned}
$$

Similarly,

$$
\begin{aligned}
\frac{\partial d_{c c}(\xi)}{\partial y_{j}} & =\frac{\partial}{\partial y_{j}}\left(\frac{\theta}{\sin \theta}\|z\|\right)=\frac{y_{j}}{\|z\|} \cdot \frac{\theta}{\sin \theta}+\|z\| \cdot \frac{\sin \theta-\theta \cos \theta}{\sin ^{2} \theta} \cdot \frac{\partial \theta}{\partial y_{j}} \\
& =\frac{y_{j}}{\|z\|} \cdot \cos \theta \\
\frac{\partial d_{c c}(\xi)}{\partial t} & =\frac{\partial}{\partial t}\left(\frac{\theta}{\sin \theta}\|z\|\right)=\|z\| \cdot \frac{\sin \theta-\theta \cos \theta}{\sin ^{2} \theta} \cdot \frac{\partial \theta}{\partial t}=\frac{1}{\|z\|} \cdot \frac{\sin \theta}{2} .
\end{aligned}
$$

Thus

$$
X_{j} d_{c c}(\xi)=\left(\frac{\partial}{\partial x_{j}}+2 y_{j} \frac{\partial}{\partial t}\right) d_{c c}(\xi)=\frac{x_{j}}{\|z\|} \cdot \cos \theta+\frac{y_{j}}{\|z\|} \cdot \sin \theta .
$$

Using equations (2.3) and (2.4), we get

$$
\begin{aligned}
X_{j} d_{c c}(\xi) & =\frac{\phi}{2 \sin \frac{\phi \rho}{2}}\left(\frac{A_{j}(1-\cos \phi \rho)+B_{j} \sin \phi \rho}{\phi} \cos \theta\right. \\
& \left.\quad+\frac{-B_{j}(1-\cos \phi \rho)+A_{j} \sin \phi \rho}{\phi} \sin \theta\right) \\
& =\left(A_{j} \sin \frac{\phi \rho}{2}+B_{j} \cos \frac{\phi \rho}{2}\right) \cos \theta+\left(-B_{j} \sin \frac{\phi \rho}{2}+A_{j} \cos \frac{\phi \rho}{2}\right) \sin \theta \\
& =A_{j} \sin \left(\frac{\phi \rho}{2}+\theta\right)+B_{j} \cos \left(\frac{\phi \rho}{2}+\theta\right) .
\end{aligned}
$$

Therefore we obtain, by (2.5),

$$
X_{j} d_{c c}(\xi)=A_{j} \sin \phi \rho+B_{j} \cos \phi \rho .
$$


On the other hand, we have, again using equations (2.3), (2.4) and (2.5),

$$
\begin{aligned}
Y_{j} d_{c c}(\xi)= & \left(\frac{\partial}{\partial y_{j}}-2 x_{j} \frac{\partial}{\partial t}\right) d_{c c}(\xi)=\frac{y_{j}}{\|z\|} \cdot \cos \theta-\frac{x_{j}}{\|z\|} \cdot \sin \theta \\
= & \frac{\phi}{2 \sin \frac{\phi \rho}{2}}\left(\frac{-B_{j}(1-\cos \phi \rho)+A_{j} \sin \phi \rho}{\phi} \cos \theta\right. \\
& \left.\quad-\frac{A_{j}(1-\cos \phi \rho)+B_{j} \sin \phi \rho}{\phi} \sin \theta\right) \\
= & \left(-B_{j} \sin \frac{\phi \rho}{2}+A_{j} \cos \frac{\phi \rho}{2}\right) \cos \theta-\left(A_{j} \sin \frac{\phi \rho}{2}+B_{j} \cos \frac{\phi \rho}{2}\right) \sin \theta \\
= & A_{j} \cos \left(\frac{\phi \rho}{2}+\theta\right)-B_{j} \sin \left(\frac{\phi \rho}{2}+\theta\right) \\
= & A_{j} \cos \phi \rho-B_{j} \sin \phi \rho .
\end{aligned}
$$

This completes the proof of Lemma 3.2.

Remark 3.3. Putting $f=u \in C_{0}^{\infty}\left(\mathbb{H}_{n}\right)$ in Lemma 3.1 and letting $R_{2} \rightarrow \infty$ and $R_{1} \rightarrow 0+$, we have

$$
|\Sigma| u(e)=-\int_{\mathbb{H}_{n}}\left\langle\nabla_{H} u, \nabla_{H} d_{c c}\right\rangle \cdot \frac{1}{d_{c c}^{Q-1}} d \xi,
$$

where $|\Sigma|=\int_{\Sigma} d \sigma$ (see 2] for an analogous representation formula associated with the Korányi-Folland nonisotropic gauge). We note the representation formula in Lemma 3.1 is also valid for $f \in C\left(\mathbb{H}_{n}\right) \cap C^{1}\left(\mathbb{H}_{n} \backslash \mathbb{Z}\right)$. In fact, since $\xi \in \mathbb{Z}$ iff $\lambda \xi \in \mathbb{Z}$ for all $\lambda>0$, one has, for all $f \in C\left(\mathbb{H}_{n}\right) \cap C^{1}\left(\mathbb{H}_{n} \backslash \mathbb{Z}\right)$, as in the proof of Lemma 3.1,

$$
\begin{aligned}
& \int_{\Sigma \backslash(\Sigma \cap \mathbb{Z})} f\left(R_{2} \xi^{*}\right) d \sigma-\int_{\Sigma \backslash(\Sigma \cap \mathbb{Z})} f\left(R_{1} \xi^{*}\right) d \sigma \\
& =\int_{\left(B_{c c}\left(e, R_{2}\right) \backslash \mathbb{Z}\right) \backslash\left(B_{c c}\left(e, R_{1}\right) \backslash \mathbb{Z}\right)} \frac{\left\langle\nabla_{H} f, \nabla_{H} d_{c c}\right\rangle}{d_{c c}^{Q-1}} d \xi,
\end{aligned}
$$

where $R_{2}>R_{1}>0$. Note that $\Sigma \cap \mathbb{Z}=\{(\mathbf{0}, 1 / \pi),(\mathbf{0},-1 / \pi)\}$. So

$$
\int_{\Sigma \backslash(\Sigma \cap \mathbb{Z})} f\left(R_{k} \xi^{*}\right) d \sigma=\int_{\Sigma} f\left(R_{k} \xi^{*}\right) d \sigma<\infty, \quad k=1,2,
$$

since $f \in C\left(\mathbb{H}_{n}\right)$. These imply that

$$
\begin{aligned}
& \left|\int_{\left(B_{c c}\left(e, R_{2}\right) \backslash \mathbb{Z}\right) \backslash\left(B_{c c}\left(e, R_{1}\right) \backslash \mathbb{Z}\right)} \frac{\left\langle\nabla_{H} f, \nabla_{H} d_{c c}\right\rangle}{d_{c c}^{Q-1}} d \xi\right| \\
& =\left|\int_{\Sigma \backslash(\Sigma \cap \mathbb{Z})} f\left(R_{2} \xi^{*}\right) d \sigma-\int_{\Sigma \backslash(\Sigma \cap \mathbb{Z})} f\left(R_{1} \xi^{*}\right) d \sigma\right| \\
& =\left|\int_{\Sigma} f\left(R_{2} \xi^{*}\right) d \sigma-\int_{\Sigma} f\left(R_{1} \xi^{*}\right) d \sigma\right|<\infty .
\end{aligned}
$$

Therefore,

$$
\int_{\left(B_{c c}\left(e, R_{2}\right) \backslash \mathbb{Z}\right) \backslash\left(B_{c c}\left(e, R_{1}\right) \backslash \mathbb{Z}\right)} \frac{\left\langle\nabla_{H} f, \nabla_{H} d_{c c}\right\rangle}{d_{c c}^{Q-1}} d \xi=\int_{B_{c c}\left(e, R_{2}\right) \backslash B_{c c}\left(e, R_{1}\right)} \frac{\left\langle\nabla_{H} f, \nabla_{H} d_{c c}\right\rangle}{d_{c c}^{Q-1}} d \xi
$$

since $\mathbb{Z}$ is a measure-zero set. This means that the representation formula in Lemma 3.1 is valid for $f \in C\left(\mathbb{H}_{n}\right) \cap C^{1}\left(\mathbb{H}_{n} \backslash \mathbb{Z}\right)$. 
Proof of Theorem 1.1. Let $\epsilon>0$. Then $0 \leq u_{\epsilon}:=\left(|u|^{2}+\epsilon^{2}\right)^{p / 2}-\epsilon^{p} \in C_{0}^{\infty}\left(\mathbb{H}_{n}\right)$. In fact, $u_{\epsilon}$ has the same support as $u$. Putting $f=u_{\epsilon} d_{c c}^{Q-p}$ in Lemma 3.1 and letting $R_{2} \rightarrow \infty$ and $R_{1} \rightarrow 0+$, we get, by Remark 3.3, since $d_{c c} \in C\left(\mathbb{H}_{n}\right) \cap C^{\infty}\left(\mathbb{H}_{n} \backslash \mathbb{Z}\right)$ and $d_{c c}(e)=0$,

$$
\int_{\mathbb{H}_{n}}\left\langle\nabla_{H} u_{\epsilon}, \nabla_{H} d_{c c}\right\rangle \cdot \frac{1}{d_{c c}^{p-1}}+(Q-p) \int_{\mathbb{H}_{n}} \frac{u_{\epsilon}}{d_{c c}^{p}}=0 .
$$

Here we use the fact that $\left|\nabla_{H} d_{c c}(\xi)\right|=1$ when $\xi \in \mathbb{H}_{n} \backslash \mathbb{Z}$ (cf. [1] ). By Hölder's inequality:

$$
\begin{aligned}
(Q-p) \int_{\mathbb{H}_{n}} \frac{u_{\epsilon}}{d_{c c}^{p}} & =-p \int_{\mathbb{H}_{n}}\left(|u|^{2}+\epsilon^{2}\right)^{(p-2) / 2} u\left\langle\nabla_{H} u, \nabla_{H} d_{c c}\right\rangle \cdot \frac{1}{d_{c c}^{p-1}} \\
& \leq p \int_{\mathbb{H}_{n}} \frac{\left(|u|^{2}+\epsilon^{2}\right)^{(p-2) / 2}|u| \cdot\left|\nabla_{H} u\right|}{d_{c c}^{p-1}} \\
& \leq p\left(\int_{\mathbb{H}_{n}} \frac{\left(|u|^{2}+\epsilon^{2}\right)^{p(p-2) / 2(p-1)}|u|^{\frac{p}{p-1}}}{d_{c c}^{p}}\right)^{\frac{p-1}{p}}\left(\int_{\mathbb{H}_{n}}\left|\nabla_{H} u\right|^{p}\right)^{\frac{1}{p}} .
\end{aligned}
$$

By dominated convergence, letting $\epsilon \rightarrow 0+$, canceling and raising both sides to the power $p$, we get (1.3). The proof of Theorem 1.1 is complete.

Remark 3.4. Let $V^{1, p}\left(\mathbb{H}_{n}\right)$ denote the space

$$
V^{1, p}\left(\mathbb{H}_{n}\right)=\left\{f \in C\left(\mathbb{H}_{n}\right) \cap C^{1}\left(\mathbb{H}_{n} \backslash \mathbb{Z}\right): \int_{\mathbb{H}_{n}} \frac{|u|^{p}}{d_{c c}^{p}}<+\infty, \int_{\mathbb{H}_{n}}\left|\nabla_{H} f\right|^{p}<+\infty\right\}
$$

In this remark, we shall show that the following Hardy inequalities hold for all $f \in V^{1, p}\left(\mathbb{H}_{n}\right)$ :

$$
\int_{\mathbb{H}_{n}}\left|\nabla_{H} f\right|^{p} \geq\left(\frac{Q-p}{p}\right)^{p} \int_{\mathbb{H}_{n}} \frac{|f|^{p}}{d_{c c}^{p}} .
$$

Furthermore, the constant $\left(\frac{Q-p}{p}\right)^{p}$ is sharp in the sense of

$$
\left(\frac{Q-p}{p}\right)^{p}=\inf _{f \in V^{1, p}\left(\mathbb{H}_{n}\right) \backslash\{0\}} \frac{\int_{\mathbb{H}_{n}}\left|\nabla_{H} f\right|^{p}}{\int_{\mathbb{H}_{n}} \frac{|f|^{p}}{d_{c c}^{p}}} .
$$

Step 1. We shall show that if $f \in V^{1, p}\left(\mathbb{H}_{n}\right)$, then

$$
\lim _{R \rightarrow+\infty} R^{Q-p} \int_{\Sigma}\left|f\left(R \xi^{*}\right)\right|^{p} d \sigma=0
$$


We note from Lemma 3.1 that

$$
\begin{aligned}
& \left.\left|R_{2}^{Q-p} \int_{\Sigma}\right| f\left(R_{2} \xi^{*}\right)\right|^{p} d \sigma-R_{1}^{Q-p} \int_{\Sigma}\left|f\left(R_{1} \xi^{*}\right)\right|^{p} d \sigma \mid \\
& =\left|\int_{B_{c c}\left(e, R_{2}\right) \backslash B_{c c}\left(e, R_{1}\right)}\left\langle\nabla_{H}\left(|f|^{p} d_{c c}^{Q-p}\right), \nabla_{H} d_{c c}\right\rangle \cdot \frac{1}{d_{c c}^{Q-1}} d \xi\right| \\
& \leq p \int_{B_{c c}\left(e, R_{2}\right) \backslash B_{c c}\left(e, R_{1}\right)} \frac{|f|^{p-1}\left|\nabla_{H} f\right|}{d_{c c}^{p-1}} d \xi+(Q-p) \int_{B_{c c}\left(e, R_{2}\right) \backslash B_{c c}\left(e, R_{1}\right)} \frac{|f|^{p}}{d_{c c}^{p}} d \xi \\
& \leq p\left(\int_{B_{c c}\left(e, R_{2}\right) \backslash B_{c c}\left(e, R_{1}\right)} \frac{|f|^{p}}{d_{c c}^{p}}\right)^{\frac{p-1}{p}}\left(\int_{B_{c c}\left(e, R_{2}\right) \backslash B_{c c}\left(e, R_{1}\right)}\left|\nabla_{H} f\right|^{p}\right)^{\frac{1}{p}} \\
& \quad+(Q-p) \int_{B_{c c}\left(e, R_{2}\right) \backslash B_{c c}\left(e, R_{1}\right)} \frac{|f|^{p}}{d_{c c}^{p}} d \xi .
\end{aligned}
$$

Thus,

$$
\left.\left|R_{2}^{Q-p} \int_{\Sigma}\right| f\left(R_{2} \xi^{*}\right)\right|^{p} d \sigma-R_{1}^{Q-p} \int_{\Sigma}\left|f\left(R_{1} \xi^{*}\right)\right|^{p} d \sigma \mid \rightarrow 0
$$

as $R_{1}, R_{2} \rightarrow+\infty$. This shows that $R^{Q-p} \int_{\Sigma}\left|f\left(R \xi^{*}\right)\right|^{p} d \sigma$ converges to a finite constant $f_{\infty} \geq 0$ as $R \rightarrow+\infty$. One must have $f_{\infty}=0$ for

$$
\int_{\mathbb{H}_{n}} \frac{|f|^{p}}{d_{c c}^{p}} d \xi=\int_{0}^{\infty} \int_{\Sigma}\left|f\left(\lambda \xi^{*}\right)\right|^{p} \lambda^{Q-p-1} d \sigma d \lambda=\int_{0}^{\infty} \frac{\lambda^{Q-p} \int_{\Sigma}\left|f\left(\lambda \xi^{*}\right)\right|^{p} d \sigma}{\lambda} d \lambda<+\infty .
$$

Therefore, (3.5) holds.

Step 2. Replace $f$ by $d_{c c}^{Q-p}|f|^{p}$ in Lemma 3.1 and let $R_{2} \rightarrow \infty$ and $R_{1} \rightarrow 0+$. Using the result of Step 1 and following the proof of Theorem 1.1, one can obtain the Hardy inequalities as expected.

We now show that the constant $\left(\frac{Q-p}{p}\right)^{p}$ is sharp. The proof is similar to that of Lemma 2.3 in [13]. Let

$$
f_{\varepsilon}(z, t)= \begin{cases}1, & d_{c c} \leq 1 \\ d_{c c}^{-(Q-p) / p-\varepsilon}, & d_{c c}>1\end{cases}
$$

We note that this family of functions can be approximated by the functions in $V^{1, p}\left(\mathbb{H}_{n}\right)$. In addition,

$$
\int_{\mathbb{H}_{n}} \frac{\left|f_{\varepsilon}\right|^{p}}{d_{c c}^{p}} d \xi=\frac{|\Sigma|}{Q-p}+\frac{|\Sigma|}{\varepsilon p}
$$

and

$$
\int_{\mathbb{H}_{n}}\left|\nabla_{H} f_{\varepsilon}\right|^{p} d \xi=\left(-\frac{Q-p}{p}-\varepsilon\right)^{p} \cdot \frac{|\Sigma|}{\varepsilon p} .
$$

Passing to the limit as $\varepsilon \rightarrow 0$, one can see that the constant $\left(\frac{Q-p}{p}\right)^{p}$ is sharp. We note that since the Carnot-Carathéodory distance is not differentiable in $\mathbb{Z}$, it is unknown whether $f_{\varepsilon}$, the family of functions above, can be approximated by the functions in $C_{0}^{\infty}\left(\mathbb{H}_{n}\right)$. So, we do not know whether the constant $\left(\frac{Q-p-\alpha}{p}\right)^{p}$ is sharp for all $f \in C_{0}^{\infty}\left(\mathbb{H}_{n}\right)$ in Theorem 1.1. 
Remark 3.5. Let $\alpha<Q-p$. Put $f=u_{\epsilon} d_{c c}^{Q-p-\alpha}$ in Lemma 3.1 and let $R_{2} \rightarrow \infty$ and $R_{1} \rightarrow 0+$. We have

$$
\begin{aligned}
(Q-p-\alpha) \int_{\mathbb{H}_{n}} \frac{u_{\epsilon}}{d_{c c}^{p+\alpha}} & =-p \int_{\mathbb{H}_{n}}\left(|u|^{2}+\epsilon^{2}\right)^{(p-2) / 2} u\left\langle\nabla_{H} u, \nabla_{H} d_{c c}\right\rangle \cdot \frac{1}{d_{c c}^{p+\alpha-1}} \\
& \leq p \int_{\mathbb{H}_{n}} \frac{\left(|u|^{2}+\epsilon^{2}\right)^{(p-2) / 2}|u| \cdot\left|\nabla_{H} u\right|}{d_{c c}^{p+\alpha-1}} \\
& \leq p\left(\int_{\mathbb{H}_{n}} \frac{\left(|u|^{2}+\epsilon^{2}\right)^{p(p-2) / 2(p-1)}|u|^{\frac{p}{p-1}}}{d_{c c}^{p+\alpha}}\right)^{\frac{p-1}{p}}\left(\int_{\mathbb{H}_{n}} \frac{\left|\nabla_{H} u\right|^{p}}{d_{c c}^{\alpha}}\right)^{\frac{1}{p}} .
\end{aligned}
$$

As in the proof of Theorem 1.1, we obtain the following Hardy-type inequalities with weights:

$$
\int_{\mathbb{H}_{n}} \frac{\left|\nabla_{H} u\right|^{p}}{d_{c c}^{\alpha}} \geq\left(\frac{Q-p-\alpha}{p}\right)^{p} \int_{\mathbb{H}_{n}} \frac{|u|^{p}}{d_{c c}^{p+\alpha}}, \quad \alpha<Q-p .
$$

Proof of Theorem 1.2. Notice that

$$
-\int_{\mathbb{H}_{n}} u \Delta_{H} u \cdot \frac{1}{d_{c c}^{2}}=-\frac{1}{2} \int_{\mathbb{H}_{n}} \Delta_{H} u^{2} \cdot \frac{1}{d_{c c}^{2}}+\int_{\mathbb{H}_{n}} \frac{\left|\nabla_{H} u\right|^{2}}{d_{c c}^{2}} .
$$

Through integration by parts, since $u \in C_{0}^{\infty}\left(\mathbb{H}_{n} \backslash \mathbb{Z}\right)$ and $d_{c c}$ is $C^{1}$ in $\mathbb{H}_{n} \backslash \mathbb{Z}$ (cf. [11]),

$$
-\frac{1}{2} \int_{\mathbb{H}_{n}} \Delta_{H} u^{2} \cdot \frac{1}{d_{c c}^{2}}=-\int_{\mathbb{H}_{n}}\left\langle\nabla_{H} u^{2}, \nabla_{H} d_{c c}\right\rangle \cdot \frac{1}{d_{c c}^{3}} d \xi .
$$

Putting $f=u^{2} d_{c c}^{Q-4}$ in Lemma 3.1 and letting $R_{2} \rightarrow \infty$ and $R_{1} \rightarrow 0+$, we have

$$
-\int_{\mathbb{H}_{n}}\left\langle\nabla_{H} u^{2}, \nabla_{H} d_{c c}\right\rangle \cdot \frac{1}{d_{c c}^{3}} d \xi=(Q-4) \int_{\mathbb{H}_{n}} \frac{u^{2}}{d_{c c}^{4}} d \xi .
$$

Thus, for all $\varepsilon>0$,

$$
\begin{aligned}
-\int_{\mathbb{H}_{n}} u \Delta_{H} u \cdot \frac{1}{d_{c c}^{2}} & =\int_{\mathbb{H}_{n}} \frac{\left|\nabla_{H} u\right|^{2}}{d_{c c}^{2}}+(Q-4) \int_{\mathbb{H}_{n}} \frac{u^{2}}{d_{c c}^{4}} d \xi \\
& \leq\left(\int_{\mathbb{H}_{n}} \frac{u^{2}}{d_{c c}^{4}}\right)^{\frac{1}{2}}\left(\int_{\mathbb{H}_{n}}\left|\Delta_{H} u\right|^{2}\right)^{\frac{1}{2}} \\
& \leq \frac{\varepsilon}{2} \int_{\mathbb{H}_{n}} \frac{u^{2}}{d_{c c}^{4}}+\frac{1}{2 \varepsilon} \int_{\mathbb{H}_{n}}\left|\Delta_{H} u\right|^{2} .
\end{aligned}
$$

Choosing $\varepsilon=\frac{Q(Q-4)}{4}$ in (3.7), we have

$$
\int_{\mathbb{H}_{n}}\left|\Delta_{H} u\right|^{2}+\frac{Q(Q-4)^{2}(Q-8)}{16} \int_{\mathbb{H}_{n}} \frac{u^{2}}{d_{c c}^{4}} \geq \frac{Q(Q-4)}{2} \int_{\mathbb{H}_{n}} \frac{\left|\nabla_{H} u\right|^{2}}{d_{c c}^{2}} .
$$

Since (see (3.6))

$$
\int_{\mathbb{H}_{n}} \frac{\left|\nabla_{H} u\right|^{2}}{d_{c c}^{2}} \geq \frac{(Q-4)^{2}}{4} \int_{\mathbb{H}_{n}} \frac{u^{2}}{d_{c c}^{4}}, \quad n \geq 2,
$$

we obtain, by (3.8),

$$
\int_{\mathbb{H}_{n}}\left|\Delta_{H} u\right|^{2} \geq\left(\frac{Q(Q-4)}{4}\right)^{2} \int_{\mathbb{H}_{n}} \frac{u^{2}}{d_{c c}^{4}} .
$$


When $Q \geq 8$, i.e. $n \geq 3$, again using (3.7) and (3.8), we obtain

$$
\frac{Q(Q-4)}{2} \int_{\mathbb{H}_{n}} \frac{\left|\nabla_{H} u\right|^{2}}{d_{c c}^{2}} \leq \int_{\mathbb{H}_{n}}\left|\Delta_{H} u\right|^{2}+\frac{Q(Q-8)}{4} \int_{\mathbb{H}_{n}} \frac{\left|\nabla_{H} u\right|^{2}}{d_{c c}^{2}} .
$$

The desired result follows.

Proof of Theorem 1.3. As in the proof of Theorem 1.1, putting $f=\frac{u_{\epsilon} d_{c c}^{Q-1}}{\left(\rho-d_{c c}\right)^{p-1}}$ in Lemma 3.1 and letting $R_{2}=\rho$ and $R_{1} \rightarrow 0+$, we get

$$
\begin{aligned}
(Q-1) & \int_{B_{c c}(e, \rho)} \frac{u_{\epsilon}}{d_{c c}\left(\rho-d_{c c}\right)^{p-1}}+(p-1) \int_{B_{c c}(e, \rho)} \frac{u_{\epsilon}}{\left(\rho-d_{c c}\right)^{p}} \\
& =-p \int_{B_{c c}(e, \rho)}\left(|u|^{2}+\epsilon^{2}\right)^{(p-2) / 2} u\left\langle\nabla_{H} u, \nabla_{H} d_{c c}\right\rangle \cdot \frac{1}{\left(\rho-d_{c c}\right)^{p-1}} \\
& \leq p \int_{B_{c c}(e, \rho)} \frac{\left(|u|^{2}+\epsilon^{2}\right)^{(p-2) / 2}|u| \cdot\left|\nabla_{H} u\right|}{\left(\rho-d_{c c}\right)^{p-1}} \\
& \leq p\left(\int_{B_{c c}(e, \rho)} \frac{\left(|u|^{2}+\epsilon^{2}\right)^{p(p-2) / 2(p-1)}|u|^{\frac{p}{p-1}}}{\left(\rho-d_{c c}\right)^{p}}\right)^{\frac{p-1}{p}}\left(\int_{B_{c c}(e, \rho)}\left|\nabla_{H} u\right|^{p}\right)^{\frac{1}{p}} .
\end{aligned}
$$

Letting $\epsilon \rightarrow 0+$, we have, by dominated convergence,

$$
\begin{aligned}
\left(\int_{B_{c c}(e, \rho)} \frac{|u|^{p}}{\left(\rho-d_{c c}\right)^{p}}\right)^{\frac{p-1}{p}} & \left(\int_{B_{c c}(e, \rho)}\left|\nabla_{H} u\right|^{p}\right)^{\frac{1}{p}} \\
\geq & \frac{p-1}{p} \int_{B_{c c}(e, \rho)} \frac{|u|^{p}}{\left(\rho-d_{c c}\right)^{p}} \\
& +\frac{Q-1}{p} \int_{B_{c c}(e, \rho)} \frac{|u|^{p}}{d_{c c}\left(\rho-d_{c c}\right)^{p-1}} \\
\geq & \frac{p-1}{p} \int_{B_{c c}(e, \rho)} \frac{|u|^{p}}{\left(\rho-d_{c c}\right)^{p}} .
\end{aligned}
$$

Canceling and raising both sides to the power $p$, we get (1.6).

\section{ACKNOWLEDGEMENT}

The authors thank the referee for a careful reading and very useful comments which improved the final version of this paper.

\section{REFERENCES}

[1] Beals R., Gaveau B. and Greiner P. C., Hamilton-Jacobi theory and the heat kernel on Heisenberg groups, J. Math. Pure Appl., 79 (2000), 633-689. MR.1776501 (2001g:35047)

[2] Cohn, W. and Lu, G. Best constants for Moser-Trudinger inequalities on the Heisenberg group, Indiana Univ. Math. J., 50 (2001), 1567-1591. MR.1889071(2003i:46032)

[3] D'Ambrosio L., Some Hardy inequalities on the Heisenberg group, Differential Equations, 40 (2004), 552-564. MR2153649 (2007d:26016)

[4] D'Ambrosio L., Hardy inequalities related to Grushin type operators, Proc. Amer. Math. Soc., 132 (2004), 725-734. MR2019949 (2005c:35050)

[5] D'Ambrosio L., Hardy-type inequalities related to degenerate elliptic differential operators, Ann. Sc. Norm. Super. Pisa Cl. Sci., (5) IV (2005), 451-486. MR2185865 (2006j:35027) 
[6] Danielli D., Garofalo N., and Phuc N. C., Inequalities of Hardy-Sobolev type in CarnotCarathéodory spaces, Sobolev Spaces in Mathematics, vol. I (dedicated to the centenary of Sergey Sobolev), International Mathematical Series, Vol. 8, Springer, 2009. MR2508841 (2010i:46056)

[7] Danielli D., Garofalo N., and Phuc N. C., Hardy-Sobolev type inequalities with sharp constants in Carnot-Carathéodory spaces, Potential Anal., 34 (2011), 223-242. MR2782971

[8] Folland G. B. and Stein E. M., Hardy spaces on homogeneous groups, Princeton University Press, Princeton, NJ, 1982. MR657581 (84h:43027)

[9] Garofalo N. and Lanconelli E., Frequency functions on the Heisenberg group, the uncertainty principle and unique continuation, Ann. Inst. Fourier (Grenoble), 40 (1990), 313-356. MR.1070830 (91i:22014)

[10] Goldstein J. A. and Zhang Q. S., On a degenerate heat equation with a singular potential, J. Funct. Anal., 186 (2001), 342-359. MR1864826 (2002k:35179)

[11] Monti R., Some properties of Carnot-Carathéodory balls in the Heisenberg group, Atti Accad. Naz. Lincei Cl. Sci. Fis. Mat. Natur. Rend. Lincei (9) Mat. Appl., 11(3) (2000), 155-167. MR.1841689(2002c:53048)

[12] Niu P., Zhang H. and Wang Y., Hardy type and Rellich type inequalities on the Heisenberg group, Proc. Amer. Math. Soc., 129 (2001), 3623-3630. MR1860496 (2002i:26009)

[13] Secchi S., Smets D., and Willem M., Remarks on a Hardy-Sobolev inequality, C. R. Acad. Sci. Paris, Ser. I, 336 (2003), 811-815. MR.1990020 (2004d:35050)

[14] Tertikas A. and Zographopoulos N. B., Best constants in the Hardy-Rellich inequalities and related improvements, Adv. Math., 209 (2007), 407-459. MR2296305(2007m:26014)

School of Mathematics and Statistics, Wuhan University, Wuhan, 430072, People's Republic of China

E-mail address: qaohyang2465@yahoo.com.cn 